\title{
A NONSINGULAR BRANS WORMHOLE: AN ANALOGUE TO NAKED BLACK HOLES
}

\author{
Amrita Bhattacharya, ${ }^{1, a}$ Ramil Izmailov, ${ }^{2, b}$ Ettore Laserra $^{3, c}$ and \\ Kamal K. Nandi $\mathbf{i}^{1,2,4, d}$ \\ ${ }^{1}$ Department of Mathematics, University of North Bengal, Raja \\ Rammohunpur, Siliguri 734 013, India \\ ${ }^{2}$ Department of Theoretical Physics, Sterlitamak State Pedagogical Academy, \\ 50, Lenin Prospect, Sterlitamak 453103, Russia \\ ${ }^{3}$ DMI, Università di Salerno, Via Ponte Don Melillo, Fisciano 84084, Salerno, \\ Italy \\ ${ }^{4}$ Joint Research Laboratory, Bashkir State Pedagogical University, 3A, \\ October Revolution Street, Ufa 450000, Russia \\ PACS numbers: 04.20.Gz, 04.62.+v \\ ${ }^{a}$ E-mail: amrita_852003@yahoo.co.in \\ ${ }^{b}$ E-mail: izmailov.ramil@gmail.com \\ ${ }^{c}$ E-mail: elaserra@unisa.it \\ ${ }^{d}$ E-mail: kamalnandi1952@yahoo.co.in
}

\begin{abstract}
In a recent paper, we showed the Jordan frame vacuum Brans Class I solution provided a wormhole analogue to Horowitz-Ross naked black hole in the wormhole range $-3 / 2<\omega<-4 / 3$. Thereafter, the solution has been criticized by some authors that, because of the presence of singularity in that solution within this range, a wormhole interpretation of it is untenable. While the criticism is correct, we show here that (i) a singularity-free wormhole can actually be obtained from Class I solution by performing a kind of Wick rotation on it, resulting into what Brans listed as his independent Class II solution (ii) the Class II solution has all the necessary properties of a regular wormhole in a revised range $-2<\omega<-3 / 2$ and finally, (iii) naked black holes, as described by Horowitz and Ross, are spacetimes where the tidal forces attain their maxima above the black hole horizon. We show that in the non-singular Class II spacetime this maxima is attained above the throat and thus can be treated as a wormhole analogue. Some related issues are also addressed.
\end{abstract}




\section{Introduction}

Lorentzian wormholes as possible astrophysical objects has been under active investigation for quite some time now. In particular, the possibility of occurrence of such objects in the Brans-Dicke theory is quite welcome since it is a natural theory that emerged as a Machian alternative 1 to Einstein's theory of general relativity. To our knowledge, regular wormhole properties of BransDicke solutions (actually, what is referred to as Class II below) are known for all $\omega<-3 / 2$ since 1973 from the work of Bronnikov [22]. Then, Agnese and La Camera [1] have shown that the Brans-Dicke scalar $\varphi$ can play the role of exotic matter provided the coupling parameter $\omega<-2$. These are followed by the work of Visser and Hochberg [2], and by the works in other classes of Brans solutions in the Jordan Frame(JF) as well as their variants in the conformally rescaled Einstein Frame (EF) $[3,4]$. The chronology stated here since the first work of Bronnikov are certainly not exhaustive but there exist many interesting articles on Brans-Dicke wormholes today (see, for instance [5-15]).

For static spherically symmetric Brans Class I solution in the $\omega=$ constant vacuum Brans-Dicke theory, we had earlier proposed a wormhole range $-3 / 2<$ $\omega<-4 / 3$ in the JF [4] and recently [15] developed a wormhole analogy to Horowitz-Ross naked black holes [16] for $\omega<-2$. The latter is the wormhole range obtained previously by Agnese and La Camera [1]. It is long known that the Class I solution (which is inversion invariant under $r \rightarrow \frac{B^{2}}{r}$ ) is plagued by curvature singularity: The $r=0$ flat spatial infinity is divided from the one at $r=\infty$ by a curvature singularity at $r=B$. (The same singularity appears in its EF version too, which is the Buchdahl solution [17]). So, the inversion transformation relates two separately singular space-times and not two regions of one connected spacetime 2 . Because of this, the Class I solution cannot be interpreted as wormhole at all, which is certainly correct, and we no longer stress on the Class I solution as a wormhole.

On the other hand, in view of the importance of Brans-Dicke theory in the competing interpretation of various astrophysical phenomena, it is important that a non-singular static spherically symmetric traversable wormhole solution in the vacuum theory be found, and the analogy in the title be confirmed. The purpose of the present paper is to achieve these.

\footnotetext{
${ }^{1}$ Since the Brans-Dicke theory contains a huge class of vacuum solutions, it might appear that the properties of the space-time would not be dictated by the presence of matter so that the theory is no longer Machian. This is not the case. The Brans-Dicke scalar $\varphi$ is always sourced by the matter stress scalar $T$, via $\left(\varphi^{; \rho}\right)_{; \rho}=\frac{T}{2 \omega+3}$. What we are doing is that we are focusing only on the matter-free region $(T=0)$, where still $G \sim\langle\varphi\rangle^{-1}$ at all space-time points. The term in vogue, namely, "vacuum Brans-Dicke theory" is a misnomer since the exterior non-trivial fields $\left(g_{\mu \nu}, \varphi\right)$ are pretty much dictated by ponderable matter. It should more properly be called the "matter-free Brans-Dicke theory". Henceforth, by vacuum we would mean matter-free. We thank an anonymous referee for raising this issue.

${ }^{2}$ We thank Professor Starobinsky for pointing this out. The Class I solution has been criticized on the basis of a "no-ghost, no-wormhole" theorem in Ref.[18] (see also [19]). However, as an aside, it might be noted that Quiros, Bonal and Cardenas [20] have shown that the cosmological singularity occurring in the EF theory is removed in the JF precisely in the range $-3 / 2<\omega \leq-4 / 3$. Quiros [21] discusses the impact of their result on the status of quantum cosmology in EF. Of course, cosmology is not our concern here.
} 
In this direction, it was shown earlier [6] that the singular Buchdahl solution in the EF could be Wick rotated into a new solution 3 , which was then identified as just the non-singular Ellis Class III wormhole [22] in the EF. In the same spirit, one would expect that the Brans Class I solution in the JF could likewise be Wick rotated into a new non-singular wormhole in the JF. This is indeed possible. The results of this paper show that the new solution (i) is just what Brans [23] listed as his independent Class II solution 4 (ii) is non-singular having all the desirable properties of a traversable wormhole but only in the revised range $-2<\omega<-3 / 2$ and (iii) confirms the wormhole analogy to naked black holes.

We point out that the main thread of development in this paper keeps only to Brans wormholes in JF, while other useful questions have been mainly addressed in the footnotes and in Sec.II, where we discuss actions in different conformal frames. In Sec. III, we outline for wider readership the salient features of a wormhole, while in Sec.IV we briefly review the singular Brans Class I solution. This solution is then used to derive a non-singular solution in Sec.V and its wormhole properties are enumerated in Sec. VI. This is followed by the argument of wormhole analogy to naked black holes in Sec. VII. Finally, Sec. VIII summarizes the contents. In the appendix, we derive wormhole solutions in the EF. We take $8 \pi G=c=1$ and a signature convention $(-,+,+,+)$.

\section{The actions}

It would be useful to see how the vacuum Brans-Dicke action in JF relates to that in the string frame and in the conformally rescaled EF5. Hence, we start from the 4-dimensional, low energy effective action of heterotic string theory compactified on a 6 -torus. The tree level string effective action in the string frame (SF or just S-frame), keeping only linear terms in the string tension $\alpha^{\prime}$ and in the curvature $\widetilde{\mathbf{R}}$, takes the following form in the matter free region $\left(S_{\text {matter }}=0\right)$ :

$$
S_{\mathrm{SF}}=\frac{1}{\alpha^{\prime}} \int d^{4} x \sqrt{-\tilde{g}} e^{-2 \tilde{\Phi}}\left[\widetilde{\mathbf{R}}+4 \tilde{g}^{\mu \nu} \tilde{\Phi}_{, \mu} \tilde{\Phi}_{, \nu}\right]
$$

where $\tilde{\Phi}$ is the massless gravidilaton field. Under the substitution $e^{-2 \tilde{\Phi}}=\varphi$, the above action reduces to the JF Brans-Dicke action with the coupling parameter $\omega$ automatically fixed to the value $\omega=-1$ :

$$
\begin{aligned}
S_{\mathrm{JF}} & =\int d^{4} x \sqrt{-\tilde{g}}\left[f(\varphi) \widetilde{\mathbf{R}}+h(\varphi) \tilde{g}^{\mu \nu} \varphi_{, \mu} \varphi_{, \nu}\right] \\
& =\int d^{4} x \sqrt{-\tilde{g}}\left[\varphi \widetilde{\mathbf{R}}-\frac{\omega}{\varphi} \tilde{g}^{\mu \nu} \varphi_{, \mu} \varphi_{, \nu}\right],
\end{aligned}
$$

\footnotetext{
${ }^{3}$ We are using the term "Wick rotation" [as in Eq.(24) below] rather loosely, which is used in quantum field theory in a very specific situation.

${ }^{4}$ Since the transition Brans I $\rightarrow$ II will be shown to be algebraically possible, albeit not by ordinary coordinate transformations, the two classes of solutions cannot really be treated as independent, contrary to the common belief. This fact was noted first by Bhadra and Sarkar [24] on the basis of the complementary transition Brans II $\rightarrow$ I.

${ }^{5}$ Two anonymous referees, respectively, have commented on the string and conformal related aspects of the Brans-Dicke theory. We address those comments here.
} 
where the gravitational coupling $f$ and the function $h$ have the forms as above 6 . This particular value of $\omega$ is actually independent of the dimensionality of the spacetime and the number of compactified dimensions $[25] 7$. When $\widetilde{\Phi}=$ constant (frozen), all the frames (1), (2) and (5) coincide.

Let us unfix $\omega$ since we are not dealing with string theory here. Under a further substitution

$$
\begin{gathered}
g_{\mu \nu}=\varphi \tilde{g}_{\mu \nu}, \\
d \phi=\sqrt{\frac{2 \omega+3}{2 \mu}} \frac{d \varphi}{\varphi}, \quad \alpha \neq 0,
\end{gathered}
$$

the action (2) goes into the action in the Einstein frame (EF) or E-frame,

$$
S_{\mathrm{EF}}=\int d^{4} x \sqrt{-g}\left[\mathbf{R}-\mu g^{\mu \nu} \phi_{, \mu} \phi_{, \nu}\right],
$$

where we have introduced a constant arbitrary parameter $\mu(= \pm 1)$ that can have any sign. The field equations from action (5) are given by

$$
R_{\mu \nu}=\mu \phi_{, \mu} \phi_{, \nu}
$$

\footnotetext{
${ }^{6} \mathrm{An}$ anonymous referee has raised an interesting question in connection with reconciling the results obtained in this paper with those in Ref.[19]. From the latter, it follows that no stable wormhole solution can exist in scalar-tensor theories with a ghost behaviour: Wormhole solutions may exist, but with a negative gravitational coupling $f(\varphi)=\varphi$ at least in some regions of the space leading to instabilities. The solution discussed in the present paper does imply such ghost region since $-2<\omega<-3 / 2$ corresponds in the Einstein frame to a scalar field with the "wrong" sign before the kinetic term. Further, wormhole existence requires, by Ref.[19], that $f(\varphi)=\varphi<0$, which can be ensured by assuming that the constant $\varphi_{0}$ in Eq.(29) below be negative. Since there is no transition (because of the exponential function there) from a positive to a negative value of the gravitational coupling, the referee speculatively suggests that the wormhole solution [Eqs.(26)-(30) below] could be stable. Intuitively, we do think that the instability could appear due to such a transition and since there is no transition in our solution, it may well represent a stable wormhole. The authors of Ref.[19] consider that the instability appears due to a negative pole of the effective potential at the transition surface to $f(\varphi)<0$. They state that this pole still does not guarantee instability, and further studies are necessary. We note that $f(\varphi)=\varphi$ is a Jordan frame variable in the action (2), and we have not considered any effective potential. Therefore, the answer is not really immediate to us. On the other hand, in the Einstein frame, $\mu=-1$ ("wrong sign") is a necessary condition. In this frame (and without any potential term), Gonzalez et al [39] analyzed the linear and nonlinear evolution of static, spherically symmetric wormhole solutions for a massless ghost scalar field. They showed that all the solutions are unstable with respect to linear and nonlinear spherically symmetric perturbations and that the perturbation causes the wormholes to either decay to a Schwarzschild black hole or undergo a rapid expansion. However, only the zero mass wormhole could be stable, as argued by Armendáriz-Picón [27]. The advantage with the Einstein frame is that the equations are far simpler and hence convenient. Eventually, the results concerning (in)stability in the Einstein frame have to be mapped back into the Jordan frame to verify the extent to which those results hold true. This requires a separate investigation.
}

${ }^{7}$ Lidsey et al [25] define the Brans-Dicke scalar not as $\varphi$ but as $e^{-2 \tilde{\Phi}}$, showing that SF and $\mathrm{JF}$ are equivalent, with the metric part remaining unaltered. They show that, more generally, $\omega=-1+\frac{1}{d}$ where $d$ is the dimension of the compactified torus on which gravidilation fields are zero. Also they argue that $\omega$ is bounded by $-1 \leq \omega<0$, where the lower bound corresponds to the value that arises in the string effective action and is formally saturated in the limit $d \rightarrow \infty$. From this viewpoint the string value, $\omega=-1$, is a fixed-point under further dimensional reduction by a finite number of dimensions. 


$$
\square^{2} \phi=0 .
$$

We see that if the kinetic term $\mu g^{\mu \nu} \phi_{, \mu} \phi_{, \nu}$ in the action has an overall positive sign, the stresses satisfy all energy conditions and there are no wormholes. If however the kinetic term $\mu g^{\mu \nu} \phi_{, \mu} \phi_{, \nu}$ has an overall negative sign, all the energy conditions are violated giving rise to the possibility of wormhole solutions. The situation is not as straightforward in the JF. Some energy conditions can be locally violated in the JF even though there are no violations (i.e., no ghost) in the EF $[18,19]$.

The action (5) is also called the string action in the EF (see Gasperini and Veneziano [26]). It seems remarkable that, starting from a general Lagrangian and imposing only wormhole constraints (mainly the constraint of energy violation), Armendáriz-Picón [27] derived the action (5) as the simplest action for a general class of microscopic scalar fields. His arguments have nothing to do with string theory, yet the end result is quite the same. So we have here a hierarchy in which the physics of dilatonic gravity meets that of wormholes.

From Eq.(4), it is obvious that the values of $\omega$ and $\mu$ can be chosen independently. In the context of string theory, we must use only the model independent, unique string value $\omega=-1$ [25]. In this case, we have $d \phi=(1 / \sqrt{2 \mu})(d \varphi / \varphi)$, and the sign of $\mu$ is essentially left undetermined by the string theory field equations in the EF, viz., Eqs.(6) and (7). What actually determines the sign of $\mu$ is the condition for energy violations by the $\phi$ - field stresses if we want wormholes to exist. The violations are ensured by an overall negative sign before the stress tensor, which can appear if $\phi$ is chosen as real function and $\mu=-1$. Alternatively, if $\phi$ is chosen to be imaginary, then $\mu=+1$. Either choice leads to a negative sign before the kinetic term $\mu g^{\mu \nu} \phi_{, \mu} \phi_{, \nu}$. However, no matter what the sign of $\mu$ is, we can always move between the string actions phrased in SF and EF respectively. Thus if there is a nonsingular wormhole in one, it can be translated into the other frame.

As correctly concluded by Flanagan [28] using the most general framework, classical observables are independent of the choice of different conformal frames. But, since the vacuum JF action (2) (with any $\omega$ ) is essentially incomplete because the matter part is absent, it has no conformal freedom due to the fact that a conformal transformation does not keep track of the matter part of the action. The vacuum theory itself may nevertheless be expressed in different conformal frame 8 but, as pointed out by Flanagan quoting Brans [31], only

\footnotetext{
${ }^{8}$ The class of conformal frames discussed by Cho [29] and Faraoni [30] is a special case of the most general action analyzed by Flanagan [28]. Faraoni [30] considered the conformal transformations $\bar{g}_{\mu \nu}=\varphi^{2 \xi} \widetilde{g}_{\mu \nu}, \sigma=\varphi^{1-2 \xi}$ under which the vacuum JF action (2) transfers to the same form: $S_{\mathrm{JF}} \rightarrow \bar{S}_{\mathrm{JF}}=\int d^{4} x(-\bar{g})^{1 / 2}\left[\sigma \overline{\mathbf{R}}-\bar{\omega} \sigma^{-1} \bar{g}^{\mu \nu} \sigma_{, \mu} \sigma_{, \nu}\right]$ with $\bar{\omega}=\frac{\omega-6 \xi(\xi-1)}{(1-2 \xi)^{2}}$, where $\xi$ is an arbitrary real constant. Lidsey et al [25] have provided a higher dimensional generalization of this transfer, calling it conformal invariance. Although there is a shift, $\left(\widetilde{g}_{\mu \nu}, \omega, \varphi\right) \rightarrow\left(\bar{g}_{\mu \nu}, \bar{\omega}, \sigma\right)$ between frames, the action itself is a scalar quantity so that the physical content of the theory in any individual frame remains the same. Note that Flanagan's general action is also form invariant [see her Eqs. (2.2) $\rightarrow$ (2.6)] in exactly the same sense as above, when all the relevant functions are suitably shifted. What essentially remain conformal frame independent are the physical predictions, as stressed by Lidsey et al
} 
one of those is a "physically correct frame". So, which one could it possibly be?

It is exactly in this context that the "right frame" proposed by Gasperini and Veneziano [26] becomes relevant. In discussing pre- and post-big bang cosmology, they go from vacuum string action (1) directly to (5) bypassing the intermediate $\omega=-1 \mathrm{JF}$ action (2) [their Eqs. (1.3) and (1.4)] and conclude that different classes of solutions do not correspond to different models of pre-big bang inflation, but simply to different kinematical representations of the same scenario in two different conformal frames. They show that different frames are just related by a local (field dependent) conformal transformation of the metric - no physical observable should depend on it. This notwithstanding, they show that there are important kinematical differences in different frames. Inflation in the S-frame, for instance, can be represented as gravitational collapse in the E-frame. In any case, they conclude that the S-frame (whose metric coincides with the sigma model metric to which fundamental strings are directly coupled) is the right frame because this is the one offering the simplest intuitive picture of how things evolve and work.

Despite the logical gap between the argument of physical correctness and simplicity, as far as frame independence of physical observables is concerned, Flanagan's conclusion is well supported in the cosmological context with the SF being favored on grounds of simplicity. We also take this standpoint in the local wormhole gravity as well, only replacing SF by JF (the frames are equivalent, as implied in [25], [26]). Note also that the 1962 list of exact Brans' solutions have been proposed only in the vacuum JF and solar gravity experiments have long been physically interpreted in this frame (see footnote 8). Since wormholes have not been ruled out, their interpretation in JF should likewise be valid. In view of these arguments, we shall assume JF as the single "physically correct frame" in the vacuum case since there is no conformal frame freedom here [28]. Certainly we are not stretching this assumption to conclude that JF is synonymous with physical frame even when matter part is involved 9 .

We shall also show in the Appendix that the wormhole interpretation need not essentially rely on the JF: The non-singular Class II wormhole in JF can be mapped to a similar non-singular wormhole in the EF so that wormhole geometry becomes conformal-frame-independent in these two frames. It would be nice to have a general proof of this statement covering arbitrary conformal frames. This concludes our discussion about conformal frames. Before we derive the non-singular Brans wormhole out of Brans Class I solution, it will be useful to provide an outline of what a wormhole is.

\section{Outline of wormhole geometry}

To be more informative, we define what a wormhole is, without going into a full scale reporting. The notions stated in this brief review will be used later. By

[25], Gasperini \& Veneziano [26] and Flanagan [28]. For instance, the string value $\omega=-1$ is a model independent prediction, which remains the same in any individual conformal frame.

${ }^{9}$ We have to point out that the question which frame, Einstein or Jordan, is more physical has a long and controversial story and we do not take any position here. Many authors treat Einstein frame as more of a mathematical convenience. 
definition, a wormhole is a topological short-cut tunnel connecting two distant regions of a single spacetime or even two universes. The spacetime has to satisfy certain constraints to qualify as a wormhole. We shall state these constraints using the Morris and Thorne [32] canonical form for the spacetime metric in "standard" coordinates, which is given by

$$
d \tau^{2}=-e^{2 \Phi(R)} d t^{2}+\left[1-\frac{b(R)}{R}\right]^{-1} d R^{2}+R^{2}\left(d \theta^{2}+\sin ^{2} \theta d \psi^{2}\right),
$$

where $\Phi(R)$ and $b(R)$ are redshift and shape functions, respectively, and $R$ is defined by the positive circumferential radius $2 \pi R$. The terminology for $\Phi$ is self-evident. The space is spherical 3D at fixed time $t$. So without loss of information, we concentrate on the $\theta=\pi / 2, t=$ constant $2 \mathrm{D}$ slice in which the metric reads

$$
d \tau^{2}=\left[1-\frac{b(R)}{R}\right]^{-1} d R^{2}+R^{2} d \psi^{2}
$$

We then remove the slice and embed it in the Euclidean 3D space having the metric

$$
d \tau^{2}=\left[1+\left(\frac{d z}{d R}\right)^{2}\right] d R^{2}+R^{2} d \psi^{2} .
$$

The isometry between (9) and (10) gives the shape of the axially symmetric embedding surface $z=z(R)$ obtained by integrating

$$
\frac{d z}{d R}= \pm\left[\frac{R}{b(R)}-1\right]^{-1 / 2} .
$$

The reason why $b(R)$ is called shape function is now clear. However, in most natural situations and certainly in the Brans solutions, the integration does not yield expressions $z=z(R)$ in a closed form. One would need to plot the shape only by numerical calculation.

The basic constraints to be satisfied by a spacetime to qualify as a wormhole are as follows:

(a) The spacetime must have two asymptotically flat regions (mouths).

(b) The mouths must be connected by a throat defined by the minimum circumferential radius. This occurs at a place $R=R_{0}$ where the vertical slope $\frac{d z}{d R}=\infty$. This implies that the throat radius $R=R_{0}$ is a root of the equation $b\left(R_{0}\right)=R_{0}$. The wormhole has a hole of finite non-zero radius $R_{0}>0$, unlike a black hole center with zero radius. Thus, $R \in\left[R_{0},+\infty\right)$.

(c) The shape function must satisfy: $b(R) / R \rightarrow 0$ as $R \rightarrow \infty$. Also, $b(R) / R \leq 1$ for all $R \geq R_{0}$.

In order that the wormhole indeed flares out to two asymptotically flat space times, the following condition

$$
\frac{d^{2} R}{d z^{2}}=\frac{b-b^{\prime} R}{2 b^{2}}>0
$$


must be satisfied at or near the throat. This inequality imposes a constraint on the type of source stress tensor $\mathbf{T}_{\mu \nu}$. Assuming an isotropic scalar field stress tensor $\mathbf{T}_{\widehat{\mu} \widehat{\nu}}^{(\varphi)}=\left[\rho, p_{R}, p_{\theta}, p_{\psi}\right]$ in the static local orthonormal frame $(\wedge)$, the left hand side of vacuum Brans-Dicke field equations $\mathbf{R}_{\widehat{\mu} \widehat{\nu}}-\frac{1}{2} \eta_{\widehat{\mu} \widehat{\nu}} \mathbf{R}=\mathbf{T}_{\widehat{\mu} \widehat{\nu}}^{(\varphi)}$ straightforwardly yield

$\rho=b^{\prime} / R^{2}, p_{R}=\left[2(R-b) \Phi^{\prime}-b / R\right] / R^{2}, p_{\theta}=p_{\psi}=p_{R}+(R / 2)\left[\left(\rho+p_{R}\right) \Phi^{\prime}+p_{R}^{\prime}\right]$,

where primes denote differentiation relative to radius $R, \rho$ is the scalar field energy density, $p_{R}$ is the radial tension, $p_{\theta}, p_{\psi}$ are transverse pressures, and $\eta_{\widehat{\mu} \widehat{\nu}}$ is the Minkowski metric in the orthonormal frame. Then the inequality in (12) can be nicely rephrased by using the Morris and Thorne [32] function $\zeta$ defined by $\zeta=-\frac{\rho+p_{R}}{|\rho|}$. Putting in it the expressions from (13), combining it with the equality in (12) and noting that $(R-b) \Phi^{\prime} \rightarrow 0$ at $R=R_{0}$, we get at the throat the following result

$$
\zeta=\frac{2 b^{2}}{R\left|b^{\prime}\right|} \frac{d^{2} R}{d z^{2}}=-\left(\frac{\rho+p_{R}}{|\rho|}\right),
$$

which shows that the flaring out condition (12) is satisfied only if $\rho+p_{R}<0$. This violates a known energy condition since, for normal matter, $\rho+p_{R}>0$ (null energy condition). A fast Lorentz boosted traveller might see the violation as $\rho<0$, which means a violation of the weak energy condition. Such energy condition violating matter is called "exotic". Thus the flaring out constraint is:

(d) We must have: $\rho<0$ and/or $\rho+p_{R}<0$ at least at or near the throat.

(e) There should be no horizon, that is, the redshift function $\Phi$ must be finite everywhere to prevent infinite redshift of signals from the traveller to outside stationary observer.

(f) The tidal forces (that are proportional to curvature tensor) experienced by a traveller finite throughout the trip.

These are the main constraints to be satisfied if a given spacetime has to represent a regular wormhole traversable in principle. Practical traversability by humans requires further that tidal forces be tolerable, that is, of the order of one Earth gravity.

IV. Singular Brans I solution

The field equations obtained by varying the vacuum JF action (2) are (dropping tilde)

$$
\begin{gathered}
\mathbf{R}_{\mu \nu}-\frac{1}{2} g_{\mu \nu} \mathbf{R}=\frac{\omega}{\varphi^{2}}\left[\varphi_{, \mu} \varphi_{, \nu}-\frac{1}{2} g_{\mu \nu} \varphi_{, \sigma} \varphi^{, \sigma}\right]+\frac{1}{\varphi}[\varphi, \mu ; \nu \\
\left.-g_{\mu \nu} \square^{2} \varphi\right], \\
\square^{2} \varphi=0 .
\end{gathered}
$$

The general solution of these field equations, in isotropic coordinates $(t, r, \theta, \psi)$, is taken in the form

$$
d \tau^{2}=-e^{2 \alpha(r)} d t^{2}+e^{2 \beta(r)}\left[d r^{2}+r^{2}\left(d \theta^{2}+\sin ^{2} \theta d \psi^{2}\right)\right] .
$$


The Brans class I solution [23] is given by

$$
\begin{gathered}
e^{\alpha(r)}=e^{\alpha_{0}}\left[\frac{1-B / r}{1+B / r}\right]^{\frac{1}{\lambda}}, \\
e^{\beta(r)}=e^{\beta_{0}}[1+B / r]^{2}\left[\frac{1-B / r}{1+B / r}\right]^{\frac{\lambda-C-1}{\lambda}}, \\
\varphi(r)=\varphi_{0}\left[\frac{1-B / r}{1+B / r}\right]^{\frac{C}{\lambda}}, \\
\lambda^{2} \equiv(C+1)^{2}-C\left(1-\frac{\omega C}{2}\right)>0,
\end{gathered}
$$

where $\lambda, \alpha_{0}, \beta_{0}, B, C$, and $\varphi_{0}$ are real constants, and the radial marker $r \in$ $(-\infty,+\infty)$. The constants $\alpha_{0}$ and $\beta_{0}$ are determined by asymptotic flatness at $r=+\infty$ as $\alpha_{0}=\beta_{0}=0$. The negative $r$-side needs a bit of explanation. Note that the metric is invariant under inversion $\bar{r} \rightarrow \frac{1}{r}$ for even values of the exponents determined by $C(\omega)$, hence $\bar{r}=0$ is a second asymptotically flat region. Defining a new coordinate chart there by $\bar{r} \rightarrow \frac{1}{r}$, which of course preserves the metric, we find that $\widehat{r} \rightarrow-\infty$ represents the second asymptotically flat region 10 Unfortunately, the two asymptotically flat spacetimes on either side are disconnected by the singularity at $r=\widehat{r}=B$, hence the solution cannot be accepted as a wormhole, as mentioned before.

To see the naked singularity, it is enough to consider the Lorentz boost invariant component of Riemann curvature in the freely falling orthonormal frame $\left(\widehat{e}_{0^{\prime}}, \widehat{e}_{1^{\prime}}, \widehat{e}_{2^{\prime}}, \widehat{e}_{3^{\prime}}\right)$, which turns out to be

$$
\mathbf{R}_{\widehat{1^{\prime} \widehat{0^{\prime}} \widehat{1}^{\prime} \widehat{0}^{\prime}}}=\frac{4 B r^{3} Z^{2}\left[\lambda\left(r^{2}+B^{2}\right)-B r(C+2)\right]}{\lambda^{2}\left(r^{2}-B^{2}\right)^{4}},
$$

\footnotetext{
${ }^{10}$ The following solution is the EF counterpart of the singular Brans Class I solution (18)(21): $d \tau_{\mathrm{EF}}^{2}=-\left(\frac{1-\frac{m}{2 r}}{1+\frac{m n}{2 r}}\right)^{2 \beta} d t^{2}+\left(1-\frac{m}{2 r}\right)^{2(1-\beta)}\left(1+\frac{m}{2 r}\right)^{2(1+\beta)}\left[d r^{2}+r^{2} d \theta^{2}+r^{2} \sin ^{2} \theta d \psi^{2}\right]$, $\phi(r)=\sqrt{\frac{2\left(1-\beta^{2}\right)}{\mu}} \ln \left[\frac{1-\frac{m}{2 r}}{1+\frac{m}{2 r}}\right]$, where $\mu, m$ and $\beta$ are arbitrary positive constants. This is known as Buchdahl solution [17], rediscovered later as the singular Ellis I solution [22]. The metric is invariant in form under inversion (for integer $\beta$ ) of the radial coordinate $r \rightarrow \frac{m^{2}}{4 r}$ and we have two asymptotically flat regions (at $r=0$ and $r=\infty$ ), the minimum circumferential radius (throat) occurring at $r_{0}=\frac{m}{2}\left[\beta+\sqrt{\beta^{2}-1}\right]$. The reality of the wormhole throat is guaranteed by $\beta^{2}>1$, but this condition also yields a naked singularity at $r=m / 2$. For $\beta=1$, the solution reduces to the Schwarzschild black hole. With regard to coordinate patches, one must make a choice as to whether the isotropic coordinate lies in the range $r \in(m / 2, \infty)$ or $r \in(0, m / 2)$. Though the patches are disconnected, both separately are homeomorphic to the standard coordinate $R \in(2 m, \infty)$ and provide equivalent coverings, each with one asymptotically flat end $(r \rightarrow 0$ and $r \rightarrow+\infty)$, of the region $R>2 m$ of the standard Buchdahl line element, variantly called Janis-Newman-Winnicour line element. The same conclusions hold in the JF as well, with $m / 2=B^{\prime}$. We thank a referee for clarifying the coordinate patches.
} 
where

$$
Z \equiv\left(\frac{r-B}{r+B}\right)^{(C+1) / \lambda} .
$$

Clearly, $\mathbf{R}_{\widehat{1}^{\prime} \widehat{0}^{\prime} \widehat{1}^{\prime} \widehat{0}^{\prime}} \rightarrow \infty$ as $r \rightarrow B$. All curvature invariants also exhibit this singular behavior. The Brans Class I solution was already investigated in detail in [15], and criticized in [18], so no need to repeat them here. We have still put the solution in view because we would need it to generate a non-singular wormhole, which we do below.

\section{Non-singular Brans wormhole}

To obtain the non-singular solution, we need to first remove the above mentioned singularity from the Class I solution. We can do it by the following operations on it:

$$
r \rightarrow \frac{1}{r^{\prime}}, B \rightarrow \frac{i}{B^{\prime}}, \lambda \rightarrow-i \Lambda, \alpha_{0} \rightarrow \epsilon_{0}, \beta_{0} \rightarrow \delta_{0}+2 \ln B^{\prime},
$$

where $B^{\prime}, \Lambda$ are real. Using the identity

$$
\tan ^{-1}(x)=\frac{i}{2} \ln \left(\frac{1-i x}{1+i x}\right),
$$

we arrive at the metric functions and the scalar field as follows

$$
d \tau^{2}=-e^{2 \alpha\left(r^{\prime}\right)} d t^{2}+e^{2 \beta\left(r^{\prime}\right)}\left[d r^{2}+r^{\prime 2}\left(d \theta^{2}+\sin ^{2} \theta d \psi^{2}\right)\right],
$$

where

$$
\begin{gathered}
\alpha\left(r^{\prime}\right)=\epsilon_{0}+\frac{2}{\Lambda} \tan ^{-1}\left(\frac{r^{\prime}}{B^{\prime}}\right) \\
\beta\left(r^{\prime}\right)=\delta_{0}-\frac{2(C+1)}{\Lambda} \tan ^{-1}\left(\frac{r^{\prime}}{B^{\prime}}\right)-\ln \left(\frac{r^{\prime 2}}{r^{\prime 2}+B^{\prime 2}}\right) \\
\varphi\left(r^{\prime}\right)=\varphi_{0} \exp \left[\frac{2 C}{\Lambda} \tan ^{-1}\left(\frac{r^{\prime}}{B^{\prime}}\right)\right] \\
\Lambda^{2} \equiv C\left(1-\frac{\omega C}{2}\right)-(C+1)^{2}>0 .
\end{gathered}
$$

Asymptotic flatness at $r^{\prime}=\infty$ requires that

$$
\epsilon_{0}=-\frac{\pi}{\Lambda}, \quad \delta_{0}=\frac{\pi(C+1)}{\Lambda} .
$$

The above solution has been listed by Brans [23] as his independent Class II solution, but we see that the two classes are not independent - one can be derived from the other by what we call Wick rotation (24). However, though not independent, Class I and II solutions are by no means equivalent as the former is singular, while the latter is regular.

Next, it is necessary to ensure that the Wick rotation does not affect the status of the new solution to be a solution of vacuum Brans-Dicke theory. This 
can be seen from the following facts: The solution (18)-(20) satisfies the field equations (15)-(16), only if the constraint (21) connecting various constants in the solution is satisfied. The transition $\lambda \rightarrow-i \Lambda$ leads to just the Brans Class II solution for which new constraint is (30), as can be found by directly putting the solution (27)-(29) in the field equations or from the Brans' 1962 list itself. Therefore the status of (27)-(29) as an exact solution of the field equations is confirmed. It should be mentioned that the wormhole interpretation does not take away its force to explain the observable predictions of usual stellar gravity. The solution in the positive mass $\left(\frac{2 B^{\prime}}{\Lambda}\right)$ side does explain the known weak field solar system predictions. 11 .

Under the radial coordinate transformation $r^{\prime} \rightarrow R$ :

$$
R=r^{\prime} \exp \left[\beta\left(r^{\prime}\right)\right]=r^{\prime}\left[1+\frac{B^{\prime 2}}{r^{\prime 2}}\right] \exp \left[\delta_{0}-\frac{2(C+1)}{\Lambda} \tan ^{-1}\left(\frac{r^{\prime}}{B^{\prime}}\right)\right],
$$

which shows that the circumferential radius $R \rightarrow \infty$ as $r^{\prime} \rightarrow \infty$, we obtain the redshift and shape functions respectively as

$$
\begin{gathered}
\Phi(R)=-\frac{\pi}{\Lambda}+\frac{2}{\Lambda} \tan ^{-1}\left[\frac{r^{\prime}(R)}{B^{\prime}}\right] \\
b(R)=R\left[1-\left\{1+\frac{2 B^{\prime}}{r^{\prime 2}(R)+B^{\prime 2}}\left(\frac{r^{\prime}(R)(C+1)}{\Lambda}-B^{\prime}\right)\right\}^{2}\right] .
\end{gathered}
$$

Using these, we shall now examine all the wormhole constraints enumerated in Sec. III.

\section{Wormhole constraints}

(a) The solution set (27)-(29) is regular everywhere including at $r^{\prime}=B^{\prime}$ as can be readily verified by computing the curvature invariants. For instance, the Riemann curvature component invariant under Lorentz boost is

$$
\mathbf{R}_{\widehat{1}^{\prime}, \widehat{0}^{\prime}, \widehat{1}^{\prime}}=-\frac{4 B^{\prime 5} r^{\prime 3}\left[\Lambda B^{\prime 2}+B^{\prime} r^{\prime}(C+2)-\Lambda r^{\prime 2}\right]}{\Lambda^{2}\left(r^{\prime 2}+B^{\prime 2}\right)^{4}} \exp \left[\frac{4(C+1)}{\Lambda} \tan ^{-1}\left(\frac{r^{\prime}}{B^{\prime}}\right)\right],
$$

\footnotetext{
${ }^{11}$ To see this, it is enough to consider the well known Eddington-Robertson expansion [33] of any metric produced by a static spherically symmetric body like the Sun: $d \tau^{2}=-\left(1-2 \alpha \frac{M}{r^{\prime}}+2 \beta \frac{M^{2}}{r^{\prime 2}}+\ldots\right) d t^{2}+\left(1-2 \gamma \frac{M}{r^{\prime}}+\ldots\right)\left[d r^{\prime 2}+r^{\prime 2}\left(d \theta^{2}+\sin ^{2} \theta d \psi^{2}\right)\right]$. PostNewtonian Schwarzschild gravity corresponds to constants $\alpha=\beta=\gamma=1$. For the Brans Class II solution (27)-(29), the mass $M=\frac{2 B}{\Lambda}$ can be identified from the Newtonian limit $e^{2 \alpha\left(r^{\prime}\right)} \simeq 1-\frac{2 M}{r^{\prime}}$. Under the same approximation, we have $C=-\frac{1}{\omega+2}$, whence the Class II solution leads to the values $\alpha=\beta=1, \gamma=\frac{\omega+1}{\omega+2}$. Currently estimated value of $\gamma$ is $\gamma$ $=2 \times(0.99992 \pm 0.00014)-1$ [34], which is close to 1 up to an accuracy of $10^{-4}$. The purpose of saying all these is that the JF is a physically correct conformal frame for the interpretation of observations in stellar gravity. As for wormholes, they are not ruled out and the corresponding observations can likewise be interpreted in JF. The important point is that the non-singular JF Class II solution yields a similar non-singular solution in the EF (see Appendix), but the latter expands on the positive mass side such that $\alpha=\beta=\gamma=1$. These are exactly the values of post-Newtonian Schwarzschild gravity with no question of Brans-Dicke coupling $\omega$ appearing anywhere. However, wormhole features still remain the same, as we will show in the Appendix.
} 
which is finite everywhere, and $\mathbf{R}_{\widehat{1}{ }^{\prime} \widehat{o}^{\prime} \widehat{1}^{\prime} \widehat{0}^{\prime}} \rightarrow 0$ when $r^{\prime} \rightarrow+\infty$ as well as when $r^{\prime} \rightarrow 0$. This second asymptotic region is evident also from the fact that $R=$ $r^{\prime} \exp \left(\beta r^{\prime}\right) \rightarrow+\infty$ as $r^{\prime} \rightarrow 012$ All the curvature invariants are also finite and go to zero in these limits. The latter limit implies that $r^{\prime}=0$ is the second asymptotically flat region. This region is seen in the metric under the transformation $r^{\prime} \rightarrow 1 / \widehat{r}$ using the identities

$$
\begin{array}{ll}
\tan ^{-1}\left(\frac{1}{x}\right) \equiv \frac{\pi}{2}-\tan ^{-1}(x), & \text { for } x>0 \\
\tan ^{-1}\left(\frac{1}{x}\right) \equiv-\frac{\pi}{2}-\tan ^{-1}(x), & \text { for } x<0,
\end{array}
$$

and then taking the limit $\widehat{r} \rightarrow-\infty$. Hence, the solution is twice asympotically flat satisfying the constraint (a).

(b) The throat occurs at the coordinate marker 13

$$
r_{0}^{\prime \pm}=B^{\prime}\left[-\frac{C+1}{\Lambda} \pm \sqrt{1+\left(\frac{C+1}{\Lambda}\right)^{2}}\right], \quad B^{\prime}>0
$$

obtained by the minimizing the circumferential radius $R=r^{\prime} \exp \left[\beta\left(r^{\prime}\right)\right]$. We shall discard the values $r_{0}^{\prime-}$ because it is always negative thereby leading to unphysical negative circumferential radius $2 \pi R_{0}^{-}$. The throat $r_{0}^{\prime+}$ is always positive because $\sqrt{1+\left(\frac{C+1}{\Lambda}\right)^{2}}>\frac{C+1}{\Lambda}$ and thus can take on any positive nonzero value leading te 14

$$
R_{0}^{+}=r_{0}^{\prime+}\left[1+\frac{B^{\prime 2}}{r_{0}^{\prime+2}}\right] \exp \left[\delta_{0}-\frac{2(C+1)}{\Lambda} \tan ^{-1}\left(\frac{r_{0}^{\prime+}}{B^{\prime}}\right)\right]>0 .
$$

It can be verified that the weak field expansions of $e^{2 \alpha\left(r^{\prime}\right)}$ using the identities (35), (36) yield respectively the asymptotic Schwarzschild masses $M^{ \pm}$

$$
\begin{aligned}
& M^{+}=\frac{2 B^{\prime}}{\Lambda} \text { as } r^{\prime} \rightarrow+\infty, \\
& M^{-}=-\frac{2 B^{\prime}}{\Lambda} \exp \left[\frac{2 \pi}{\Lambda}\right] \text { as } \widehat{r} \rightarrow-\infty
\end{aligned}
$$

\footnotetext{
${ }^{12}$ We thank an anonymous referee for correcting an error in the earlier version. This correction eventually led to a better understanding of the coordinate cover on either side of the regular wormhole. Essentially, it is a single coordinate cover but described in different notations $r^{\prime}(\rightarrow+\infty)$ and $\widehat{r}(\rightarrow-\infty)$ joined smoothly at $r^{\prime}=\widehat{r}=B^{\prime}$. Alternatively, the asymptotic region $R \in\left[R_{0},+\infty\right)$ is covered twice, once by $r^{\prime} \in\left[r_{0}^{\prime+},+\infty\right)$ and again upside down by $r^{\prime} \in\left[0, r_{0}^{\prime+}\right]$. The homeomorphism between the two covers is evident.

${ }^{13}$ We sincerely thank a referee for pointing out an error in the earlier version. There would be a negative sign before the first term in the bracket of Eq.(38), which was missing in the earlier version. However, there is no alteration in the conclusions.

${ }^{14}$ The radial coordinate $r^{\prime}$ is an abstract coordinate chart covering the entire space, whereas the Morris-Thorne radius $R$ is defined by physically measurable circumference but it does not cover the entire space. The throat radius can be calculated either by the minimal circumferential radius $2 \pi R$ or from the shape function $b(R)$. Both of course yield the same answer.
} 
of two asymmetric mouths lying on either side of the throat $r=r_{0}^{\prime+}$. Hence the contraint (b) is satisfied.

(c) It can be directly seen that the shape function satisfies: $b(R) / R \rightarrow 0$ as $R \rightarrow \infty$ and $b(R) / R \leq 1$ for all $R \geq R_{0}$. Hence (c) is satisfied.

(d) The stress components (13) can be computed from (27)-(29) giving the scalar field energy density and radial pressure as

$$
\begin{gathered}
\rho=-\frac{4 B^{\prime 6} r^{\prime 4}\left[(C+1)^{2}+\Lambda^{2}\right]}{\Lambda^{2}\left(r^{\prime 2}+B^{\prime 2}\right)^{4}} \exp \left[\frac{4(C+1)}{\Lambda} \tan ^{-1}\left(\frac{r^{\prime}}{B^{\prime}}\right)\right], \\
\rho+p_{R}=-\frac{4 B^{\prime 5} r^{\prime 3}\left[\Lambda C\left(r^{\prime 2}-B^{\prime 2}\right)+2 B^{\prime} r^{\prime}\left(C+1+\Lambda^{2}\right)\right]}{\Lambda^{2}\left(r^{\prime 2}+B^{\prime 2}\right)^{4}} \\
\quad \times \exp \left[\frac{4(C+1)}{\Lambda} \tan ^{-1}\left(\frac{r^{\prime}}{B^{\prime}}\right)\right] .
\end{gathered}
$$

It is evident that $\rho<0$ everywhere in the spacetime and so weak energy condition is violated. Hence $(d)$ is satisfied, whatever be the value of a real $\Lambda$.

Now, the weak energy condition $(\rho>0)$ is stronger than the null energy condition $\left(\rho+p_{R}>0\right)$ and the violation of the latter is proven to be also the minimal violation required of a wormhole [35]. To ensure that $\rho+p_{R}<0$ at or near the throat, we would need to fix the values of $\Lambda, C$ and $r_{0}^{\prime+}$ in terms of $\omega$. To this end, using the weak field value

$$
C=-\frac{1}{\omega+2}
$$

we get

$$
\Lambda= \pm \sqrt{-\frac{2 \omega+3}{2 \omega+4}}
$$

The condition that $\Lambda$ be real immediately yields a new range $-2<\omega<-3 / 2$. Outside this range, $\Lambda$ becomes imaginary, which in turn contradicts the Wick rotation (24). Hence those values outside the above interval for $\omega$ do not correspond to wormholes. Putting the values of $C$ and $\Lambda$, together with any value of $\omega$ in the allowed range, we would obtain two values for the throat $r_{0}^{\prime \pm}$, one positive and the other negative, for real $B^{\prime}$. The negative value is of course discarded. Figs. 1 and 2 show that both $\rho<0$ and $\rho+p_{R}<0$ in the new range $-2<\omega<-3 / 2$. Both $\rho$ and $\rho+p_{R}$ go to zero at the asymptotic ends $r^{\prime} \rightarrow+\infty$ as well as at $\widehat{r} \rightarrow-\infty$, as expected.

(e) It is evident from $\Phi$ in (33) that it is always finite everywhere including at the asymptotic limits $r^{\prime}=0$ and $\infty$. Hence (e) is satisfied.

(f) The differential of the radial tidal acceleration $\Delta a^{r}$ in the static orthonormal frame $\left(\widehat{e}_{t}, \widehat{e}_{R}, \widehat{e}_{\theta}, \widehat{e}_{\varphi}\right)$ is given by

$$
\Delta a^{r}=-\mathbf{R}_{\widehat{R} \hat{t} \widehat{R} \hat{t}} \xi^{R},
$$


where $\xi^{R}$ is the radial component of the separation vector and the curvature tensor component is given by [32]

$$
\mathbf{R}_{\widehat{R} \hat{t} \widehat{R} \hat{t}}=(1-b / R)\left\{-\Phi^{\prime \prime}+\frac{b^{\prime} R-b}{2 R(R-b)} \Phi^{\prime}-\left(\Phi^{\prime}\right)^{2}\right\} .
$$

This component is invariant under a Lorentz boost [16,32], $\mathbf{R}_{\widehat{1^{\prime}} \widehat{0^{\prime}} \widehat{\widehat{\prime}}^{\prime} \widehat{0}^{\prime}}=\mathbf{R}_{\widehat{R} \widehat{t} \widehat{R} \widehat{t}}$. For the metric given by (27)-(29), we already calculated in (35) the radial tidal acceleration $\mathbf{R}_{\hat{1}^{\prime} \widehat{0}^{\prime}, \widehat{1}^{\prime} \widehat{0}^{\prime}}$ and showed that it is always finite in the Lorentz boosted orthonormal frame $\left(\widehat{e}_{0^{\prime}}, \widehat{e}_{1^{\prime}}, \widehat{e}_{2^{\prime}}, \widehat{e}_{3^{\prime}}\right)$ of the traveller.

The lateral tidal forces in the Lorentz boosted frame are $[16,32]$

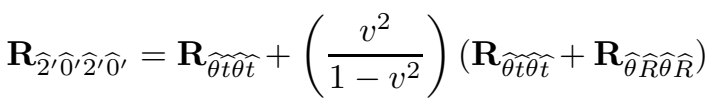

$$
\begin{aligned}
& \mathbf{R}_{\widehat{3^{\prime} \widehat{o}^{\prime}, \widehat{3}^{\prime} \widehat{0}^{\prime}}}=\mathbf{R}_{\widehat{\varphi} \widehat{\varphi} \widehat{\varphi} \widehat{t}}+\left(\frac{v^{2}}{1-v^{2}}\right)\left(\mathbf{R}_{\widehat{\varphi} \widehat{t} \widehat{\varphi} \widehat{t}}+\mathbf{R}_{\widehat{\varphi} \widehat{R} \widehat{\varphi} \widehat{R}}\right),
\end{aligned}
$$

where $v$ is the instantaneous velocity of the traveller. Since, by spherical symmetry, $\mathbf{R}_{\widehat{\theta t} \widehat{t} \widehat{\theta}}=\mathbf{R}_{\widehat{\varphi} \hat{t} \widehat{\varphi} \widehat{t}}$ and $\mathbf{R}_{\widehat{\theta} \widehat{R} \widehat{\theta} \widehat{R}}=\mathbf{R}_{\widehat{\varphi} \widehat{R} \widehat{\varphi} \widehat{R}}$, we get, for the canonical metric (8):

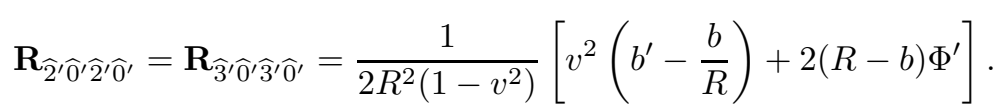

For $v<1$, they remain finite. Since all the tidal forces are finite for a trip across, the constraint $(f)$ is satisfied.

Thus the Brans Class II solution represents a regular wormhole, traversable in principle. However, the requirement of comfortable trip by humans in practice, viz., that the tidal forces be tolerable (not exceeding one Earth gravity) is a separate question. We shall discuss the relevant magnitudes in the context of analogue wormhole.

VII. Wormhole analogy

First, let us state what we mean by naked black holes. The idea was first discussed by Horowitz and Ross [16]. They defined the naked black hole as a spacetime in which an infalling observer meets the maximum tidal force not at the horizon but above it. In a freely falling frame, the curvature components could be larger than those at the horizon. Normally, tidal forces are maximum at the classically invisible horizon. Since the region of large tidal forces is visible to distant observers, Horowitz and Ross called such objects "naked black holes". In our case, the role of invisible horizon is played by the visible throat of the analogue wormhole.

Next, to see the wormhole analogy, let us assess the variation in the curvature components above the throat by explicitly calculating the components in the infalling orthonormal frame $\left(\widehat{e}_{0^{\prime}}, \widehat{e}_{1^{\prime}}, \widehat{e}_{2^{\prime}}, \widehat{e}_{3^{\prime}}\right)$. The results are:

$$
\mathbf{R}_{\widehat{1^{\prime}, \widehat{0}^{\prime}, \widehat{1}^{\prime} \widehat{0}^{\prime}}}=-\frac{4 B^{\prime 5} r^{\prime 3}\left[\Lambda B^{\prime 2}+B^{\prime} r^{\prime}(C+2)-\Lambda r^{\prime 2}\right]}{\Lambda^{2}\left(r^{\prime 2}+B^{\prime 2}\right)^{4}} \exp \left[\frac{4(C+1)}{\Lambda} \tan ^{-1}\left(\frac{r^{\prime}}{B^{\prime}}\right)\right]
$$




$$
\begin{aligned}
& \mathbf{R}_{\widehat{2}^{\prime}, \widehat{0}^{\prime} \widehat{2}^{\prime} \widehat{0}^{\prime}}=\mathbf{R}_{\widehat{3}^{\prime} \widehat{0}^{\prime} \widehat{3}^{\prime} \widehat{0}^{\prime}}=\frac{2 B^{\prime 5} r^{\prime 3} \exp \left[\frac{4(C+1)}{\Lambda} \tan ^{-1}\left(\frac{r^{\prime}}{B^{\prime}}\right)\right]}{\Lambda^{2}\left(r^{\prime 2}+B^{\prime 2}\right)^{4}\left(1-v^{2}\right)} \times \\
& {\left[\Lambda\left(r^{\prime 2}-B^{\prime 2}\right)\left\{(C+1) v^{2}-1\right\}+2 B^{\prime} r^{\prime}\left\{C+1+\Lambda^{2} v^{2}\right\}\right] . }
\end{aligned}
$$

Putting the value of $C$ and either value of $\Lambda$ in turn we can express $\left|\mathbf{R}_{\widehat{1^{\prime}}, \widehat{0}^{\prime}, \widehat{1}^{\prime}}\right|=$ $\left|g\left(\omega, r^{\prime}, B^{\prime}\right)\right|$, where the function $g$ results from the right hand side of (35). The behavior of $g$ in Fig.3 then exhibits the wormhole analogue of the naked black hole. For positive $\Lambda$, curvature increases above the throat resembling the curvature enhancement above the horizon in naked black holes. For negative $\Lambda$, there is no enhancement above the throat, as measured by the infalling observer, and hence no such analogy is possible (Fig.4).

Regardless of the implications of wormhole analogy, the regular Brans II wormhole is traversable only in principle, not in practice. To be suitable for comfortable travel by a human of length 2 meters, the radial tidal acceleration should be roughly of the order of one Earth gravity $g_{\oplus}\left(=\frac{G M_{\oplus}}{r_{\oplus}^{2}} \simeq 980 \mathrm{~cm} / \mathrm{sec}^{2}\right)$. In relativistic units, it should be $\frac{g_{\oplus}}{c^{2}}$. Since $\xi^{R}$ has the dimension $L$ of length, looking at (46), we get that the dimension of $\mathbf{R}_{\widehat{1}^{\prime}, \widehat{o}^{\prime}, \widehat{1}^{\prime}}$ must be $\frac{g_{\oplus}}{c^{2} \times L} \sim L^{-2}$. Therefore, the right hand side Eq.(28) should be less than $\lesssim \frac{g_{\oplus}}{c^{2} \times 2 \mathrm{mtrs}} \sim 10^{-20} \mathrm{~cm}^{-2}$ [32], which requires that the magnitude of the curvature components should be very close to zero in the orthonormal frame of the traveller. Such a condition is easily provided by $\Phi=0$, which is not the case here. To have an idea of the magnitudes involved, we focus on a typical value in Fig.3, say, $\omega=-1.7$ with positive $\Lambda$ and observe that $|g|=0.001 \mathrm{~cm}^{-2}$ at $r_{0}^{+}=0.17$. This is $10^{17}$ orders of magnitude more than the value is required $\left(10^{-20} \mathrm{~cm}^{-2}\right)$ for practical travel, which is too large to be comfortable with! The maximum of $|g|$ occurs up nearby, at $r^{\prime}=0.2$, where $|g|=0.002 \mathrm{~cm}^{-2}$. Though insignificant, it's still an enhancement in radial curvature over the value at $r_{0}^{+}$, supporting the analogy in principle. However, no such enhancement is observed when $\Lambda$ is negative, as shown in Fig.4.

We can see how the lateral forces fix the velocity $v$ of an inanimate particle especially at the highest curvature at $r^{\prime}=0.2$. Assuming that the radial and lateral accelerations are of the same order, we have the condition

$$
\left|\mathbf{R}_{\widehat{2^{\prime}}, \widehat{0^{\prime}}, \widehat{2}^{\prime}, \widehat{0}^{\prime}}\right| \leq 0.002 \text {. }
$$

Putting the value $\omega=-1.7$ and in units $B^{\prime}=1$, we get from (50)

$$
0.0032-0.0044 v^{2} \leq 0.002\left(1-v^{2}\right),
$$

which yields $0.59 \leq v<1$.

VIII. Summary

There has been a controversy over several years as to which frame, JF or $\mathrm{EF}$, is physical. While the aim of the present paper is not to conclude this debate, we think that physical predictions should in general be conformal-frameindependent where conformal freedom is allowed. We chose JF to work out the wormhole solution for two reasons: On the one hand, the vacuum case has no 
conformal freedom, which means that only one frame has to be physical [28], and on the other, weak field solar gravity predictions have been well interpreted in the JF [34]. In short, we adhered to the Brans-Dicke theory in the frame proposed originally by its progenitors on the basis of Machian philosophy. In the EF, the theory is no longer Machian, the parameter $\omega$ disappears leading indistinguishably to the post-Newtonian Schwarzschild values. Regardless of this, the same wormhole geometry is well preserved also in the EF (see Appendix). Therefore, the validity of our analysis need not be treated as restricted only to JF.

Now we come to the main thrust of the paper. From Sec.III downwards, we have systematically developed what constraints are to be satisfied by a spacetime to qualify as a regular wormhole. Several related important questions are addressed in the footnotes in order that a reader can smoothly follow the string of developments in the text. Thus, after reviewing the basic wormhole constraints, we have derived a regular wormhole solution from the singular Brans Class I solution. We find that the new regular solution is just the Brans Class II solution to be found in his list of independent solutions of the vacuum theory. However, since the passage Class I $\leftrightarrow$ Class II is possible via Wick rotations, one might not regard them as strictly independent solutions. This is a new information, probably unknown at large.

We have demonstrated that the regular Class II solution nicely satisfies all the constraints required of a wormhole, while preserving the observed weak field post-Newtonian values in the positive mass mouth. We see that $\rho<0$ everywhere [see Eq.(42)], no matter what the value of $\omega$ is. The bottomline is that the solution is a born wormhole for any $\omega$ as long as we don't specify $C(\omega)$ and remain happy with the violation of weak energy condition alone. But specify $C(\omega)$ we must if we want a tally with, say, solar system observations. In this case, limits on $\omega$ will appear. Accordingly, we chose the weak field equation (44), and the requirement that $\Lambda(\omega)$ be real immediately yields the range $-2<\omega<-3 / 2$. We observe an interesting thing happening in this range: The minimal violation of energy condition, viz., $\rho+p_{R}<0$, though not mandatory, is respected in addition to $\rho<0$ (see Figs.1 \& 2). It is however quite likely that the $\omega$ - range would be different for different $C(\omega)$ functions (see Ref.[10] for details on this point). Such specifications of $C(\omega)$ seem always possible under different physical circumstances, see [38].

So, all in all, we have a true Brans-Dicke wormhole with a stellar sized mouth that can act as a launching pad for passage through the throat out into the negative mass mouth. The wormhole is like a Janus-faced object that attracts matter at one end and spews out at the other. A hypothetical traveller has to accelerate to get out of the spacetime of attractive positive mass $\left(M^{+}\right)$, and after passing the throat, decelerate to come to a stop in the spacetime of repulsive negative mass $\left(M^{-}\right)$. We think that the Class II solution is a remarkable example of a natural wormhole (as distinct from artificially constructed ones, such as those in Morris and Thorne [32]) provided by the Brans-Dicke theory. If the theory is true, the gravity-scalar field coupling via $\omega$ does lead to the possibility of exotic matter being present in the spacetime. However, since 
$\Phi \neq 0$, human travel seems impossible as the tidal forces would be intolerable at or near the throat. Only an inanimate sufficiently hard test object (say, a robot) can pass through to the other side.

Finally, we have shown that there can be a wormhole analogy to HorowitzRoss naked black holes when $\Lambda>0$ (Fig.3). But this analogy is more academic than practical because the rise in curvature compared to that in the throat is too tiny. No such rise is observed when $\Lambda<0$ (Fig.4). Several questions still need to be answered. For instance, the possibility of naked black holes can impact the problem of information puzzle at the horizon, as speculated by those authors. What will be its parallel with wormholes, remembering that wormholes are not collapsed objects? How to have a significant enhancement in curvature? Will a different $C(\omega)$ valid near strong gravity [38] such as that of a neutron star do? These we reserve as tasks for the future.

\section{Acknowledgments}

We sincerely thank Professor A.A. Starobinsky for several informative correspondences. One of us (AB) thanks the CSIR, Government of India for financial support through NET-JRF fellowship. KKN acknowledges the financial support provided by the GNFM-INDAM grant of the Government of Italy under which major part of the work has been carried out at the University of Salerno. All authors are deeply indebted to Guzel N. Kutdusova and Sonali Sarkar for their moral and technical support.

\section{Figure captions}

Fig.1. We take unit as $B^{\prime}=1$, the positive sign before $\Lambda$ and a typical value in the new range $-2<\omega<-3 / 2$, say, $\omega=-1.7$. The positive value of the throat radius is $r_{0}^{++}=0.17, C=-3.33, \Lambda=0.81$ and $R_{0}^{+}=2.31$. We see that both $\rho<0$ and $\rho+p_{R}<0$ around the throat.

Fig.2. We take unit as $B^{\prime}=1$, the negative sign before $\Lambda$ and a typical value in the new range $-2<\omega<-3 / 2$, say, $\omega=-1$. . The positive value of the throat radius is $r_{0}^{\prime+}=5.88, C=-3.33, \Lambda=-0.81$ and $R_{0}^{+}=15.84$. We see that both $\rho<0$ and $\rho+p_{R}<0$ everywhere.

Fig.3. We take unit as $B^{\prime}=1$, the positive sign before $\Lambda$ and a typical value in the new range $-2<\omega<-3 / 2$, say, $\omega=-1.7$. The positive value of the throat radius is $r_{0}^{\prime+}=0.17, C=-3.33$ and $\Lambda=0.81$. The maximum value of $\mathbf{R}_{\widehat{1}^{\prime} \widehat{0}^{\prime} \widehat{1}^{\prime} \widehat{0}^{\prime}}$ occurs at $r^{\prime}=0.2$, which lies above the throat $r_{0}^{\prime+}$. The plot shows curvature enhancement away from from the throat.

Fig.4. We take unit as $B^{\prime}=1$, the negative sign before $\Lambda$ and a typical value in the new range $-2<\omega<-3 / 2$, say, $\omega=-1.7$. The positive value of the throat radius is $r_{0}^{\prime+}=5.88, C=-3.33$ and $\Lambda=-0.81$. The plot shows a steady decrease in curvature $\left|\mathbf{R}_{\widehat{1}, \widehat{0^{\prime}}, \widehat{\prime}, \widehat{0}^{\prime}}\right|$ away from the throat, hence no analogy exists in this case.

\section{Appendix \\ The EF variant of the JF Brans II solution}

In the main text, the Class II solution was interpretated as a regular wormhole only in the JF. But this is no limitation. As an illustration, we may go over to the EF to see that the same interpretation still holds as detailed below. 
After redefining the constants

$$
\frac{2 C}{\Lambda} \rightarrow 4 \lambda_{1}, \frac{2(C+2)}{\Lambda} \rightarrow 4 \gamma_{1},-\frac{2 \pi}{\Lambda} \rightarrow \epsilon_{1}, \frac{2 \pi(1+C)}{\Lambda} \rightarrow \zeta_{1},
$$

the JF solution (27)-(29) reads in the conformally rescaled EF as follows:

$$
d \tau_{\mathrm{EF}}^{2}=-P\left(r^{\prime}\right) d t^{2}+Q\left(r^{\prime}\right)\left[d r^{\prime 2}+r^{\prime 2}\left(d \theta^{2}+\sin ^{2} \theta d \psi^{2}\right)\right],
$$

where

$$
\begin{aligned}
& P\left(r^{\prime}\right)=\exp \left[2 \epsilon_{1}+4 \gamma_{1} \tan ^{-1}\left(r^{\prime} / B^{\prime}\right)\right], \\
& Q\left(r^{\prime}\right)=\left(1+\frac{B^{\prime 2}}{r^{\prime 2}}\right)^{2} \exp \left[2 \zeta_{1}-4 \gamma_{1} \tan ^{-1}\left(r^{\prime} / B^{\prime}\right)\right], \\
& \phi\left(r^{\prime}\right)=4 \lambda_{1} \tan ^{-1}\left(r^{\prime} / B^{\prime}\right), \text { where } 2 \lambda_{1}^{2}=1+\gamma_{1}^{2} .
\end{aligned}
$$

Asymptotic flatness requires that $\epsilon_{1}=-\pi \gamma_{1}$ and $\zeta_{1}=\pi \gamma_{1}$. The constraint equation $2 \lambda_{1}^{2}=1+\gamma_{1}^{2}$ among free constants comes from the EF field equations when the solution is put into them. This is the EF counterpart of the JF Class II solution. To make it look more familiar, transform the radial variable as $\ell=r^{\prime}+\frac{B^{\prime 2}}{r^{\prime}}$. Then the solution (56)-(58) goes over into

$$
\begin{aligned}
& d \tau_{\mathrm{EF}}^{2}=-F(\ell) d t^{2}+F^{-1}(\ell)\left[d \ell^{2}+\left(\ell^{2}+m^{2}\right)\left(d \theta^{2}+\sin ^{2} \theta d \psi^{2}\right)\right], \\
& F(\ell)=\exp \left[-2 \pi \gamma_{1}+4 \gamma_{1} \tan ^{-1}\left(\frac{\ell+\sqrt{\ell^{2}+m^{2}}}{m}\right)\right], \\
& \phi(\ell)=4 \lambda_{1} \tan ^{-1}\left(\frac{\ell+\sqrt{\ell^{2}+m^{2}}}{m}\right),
\end{aligned}
$$

where we have identified $m=2 B^{\prime}$ and $\ell \in(-\infty,+\infty)$. Following the same steps as in Sec.V, it can be straightaway verified that this is a twice asymptotically flat regular wormhole similar to that in the JF. So the features of wormhole geometry are conformally well preserved, as we had promised to show in Sec.II. When $m=0$, the spacetime is flat, as expected. When $m \neq 0$ but $\gamma_{1}=0$, we obtain what is known as a "zero total mass wormhole", in which the pure scalar field masses of opposite signs at either side add exactly to zero. Its lensing properties have been studied in Refs. [36,37].

The Eqs.(60), (61) are exactly the same as the regular Ellis-Bronnikov wormhole [22] in the EF already given by

$$
\begin{aligned}
& F_{\text {Ellis }}(\ell)=\exp \left[-2 \pi \gamma_{1}+4 \gamma_{1} \tan ^{-1}\left(\frac{\ell}{m}\right)\right], \\
& \phi_{\text {Ellis }}(\ell)=4 \lambda_{1} \tan ^{-1}\left(\frac{\ell}{m}\right)
\end{aligned}
$$


both the sets identically satisfying the EF field equations (6), (7):

$$
\begin{aligned}
{\left[\left(\ell^{2}+m^{2}\right) \frac{F^{\prime}}{F}\right]^{\prime} } & =0, \\
\left(\frac{F^{\prime}}{F}\right)^{2}+\frac{4 m^{2}}{\left(\ell^{2}+m^{2}\right)^{2}}-2 \phi^{2} & =0,
\end{aligned}
$$

yielding the same constraint equation $2 \lambda_{1}^{2}=1+\gamma_{1}^{2}$, where primes denote differentiation with respect to $\ell$. The zero mass case is also the same. The point we want to clarify is that the regular wormhole derived in this article need not rely exclusively on the JF but, as we have shown, the EF conformal variant yields exactly the same regular wormhole as well. The identity of solutions (60), (61) with those in (62), (63) can be easily seen by redefintion of variables and arctan identities.

However, note that the two sets of solutions have been obtained from the same JF Class I but by two different routes:

JF Class I $\stackrel{\text { conf. trans. }}{\rightarrow}$ Buchdahl $\stackrel{\text { coord. trans. }+ \text { Wick }}{\rightarrow}$ Ellis-Bronnikov wormhole (62), (63) in EF (Ref.[6]).

JF Class I $\stackrel{\text { Wick rotation }}{\rightarrow}$ JF Class II $\stackrel{\text { conf.trans.+coord.trans. }}{\rightarrow}$ New solution (60)-(61) in $\mathrm{EF}$ (this paper).

The identity of the end result shows that the operations are commutative.

References

[1] A.G. Agnese and M. La Camera, Phys. Rev. D 51, 2011 (1995).

[2] M. Visser and D. Hochberg, Proc. Haifa Workshop on the Internal Structure of Black Holes and Space Time Singularities (Jerusalem, Israel, June, 1997) [arXiv:gr-qc/970001], p.20.

[3] K.K. Nandi, A. Islam, and J. Evans, Phys. Rev. D 55, 2497 (1997).

[4] K.K. Nandi, B. Bhattacharjee, S.M.K. Alam, and J. Evans, Phys. Rev. D 57, 823 (1998).

[5] A. Bhadra, K. Sarkar, D. P. Datta, and K. K. Nandi, Mod. Phys. Lett. A 22 (2007) 367 arXiv:gr-qc/0605109.

[6] Kamal K. Nandi, Ilnur Nigmatzyanov, Ramil Izmailov and Nail G. Migranov, Class.Quant.Grav. 25,165020 (2008).

[7] Ernesto F. Eiroa, Martin G. Richarte, and Claudio Simeone, Phys. Lett. A 373, 1 (2008); ibid. A 373 E2399 (2009).

[8] Arunava Bhadra, Ion Simaciu, Kamal Kanti Nandi, and Yuan-Zhong Zhang, Phys. Rev. D 71, 128501 (2005).

[9] L.A. Anchordoqui, S. P. Bergliaffa, and D.F. Torres, Phys. Rev. D 55, 526 (1997).

[10] Francisco S. N. Lobo and Miguel A. Oliveira, Phys. Rev. D 81, 067501 (2010).

[11] J.P.S. Lemos, F.S.N. Lobo, and S.Q. de Oliveira, Phys. Rev. D 68 064004 (2003).

[12] F.S. N. Lobo, Phys. Rev. D 71, 084011 (2005); Phys. Rev. D 73, 064028 (2006). 
[13] S. V. Sushkov, Phys.Rev. D 71, 043520 (2005).

[14] Ernesto F. Eiroa and Claudio Simeone [arXiv: gr-qc/1008.0382].

[15] Amrita Bhattacharya, Ilnur Nigmatzyanov, Ramil Izmailov, and Kamal K. Nandi, Class. Quant. Grav. 26, 235017 (2009).

[16] G.T. Horowitz and S.F. Ross, Phys. Rev. D 56, 2180 (1997).

[17] H. A. Buchdahl, Phys. Rev. 115, 1325 (1959). There is a bit of history here: Bronnikov and Grinyok [Grav. \& Cosmol. 7, 297 (2001)] point out that the solution was first derived by Fisher [Zh. Eksp. Teor. Fiz. 18, 636 (1948)] for a canonical scalar and that it been independently rediscovered by Bergmann and Leipnik in 1957 (also called anti-Fisher solution) for a phantom scalar, corresponding to Brans-Dicke $\omega<-3 / 2$. Buchdahl mentions that their solution is disfigured by an inconvenient choice of coordinates.

[18] K.A. Bronnikov, M.V. Skvortsova, and A.A. Starobinsky, Grav. Cosmol.16, 216 (2010) [arXiv:gr-qc/1005.3262v1].

[19] K.A. Bronnikov and A.A. Starobinsky, JETP Lett. 85,1 (2007).

[20] Israel Quiros, Rolando Bonal, and Ronaldo Cardenas, Phys. Rev. D 62, 044042 (2000).

[21] I. Quiros, Phys. Rev. D 61, 124026 (2000)

[22] H.G. Ellis, J. Math. Phys. 14, 104 (1973); ibid. 15, 520E (1974). The solution has been independently discovered also by K.A. Bronnikov, Acta Phys. Polon. B 4, 251 (1973). We call it Ellis-Bronnikov wormhole.

[23] C. H. Brans, Phys. Rev. 125, 2194 (1962).

[24] Arunava Bhadra and Kabita Sarkar, Gen. Rel. Grav. 37, 2189 (2005).

[25] J.E. Lidsey, D. Wands, and E.J. Copeland, Phys. Rep. 337, 343 (2000) hep-th/9909061v2].

[26] M. Gasperini and G. Veneziano, Phys. Rep. 373, 1 (2003) hep-th/0207130.

[27] C. Armendáriz-Picón, Phys. Rev. D 65, 104010 (2002).

[28] É.É. Flanagan, Class. Quant. Grav. 21, 3187 (2004).

[29] Y.M. Cho, Phys. Rev. Lett. 68, 3133 (1992).

[30] V. Faraoni, Phys. Lett. A 245, 26 (1998).

[31] C.H. Brans, Class. Quant. Grav. 5, L197 (1988).

[32] M. S. Morris and K. S. Thorne, Am. J. Phys. 56, 395 (1988).

[33] S. Weinberg, Gravitation \& Cosmology, John Wiley, New York, 1972.

[34] C.M. Will, Living Rev. Relativ. 4, 2001-2004 (2001).

[35] D. Hochberg and M. Visser, Phys. Rev. Lett. 81, 746 (1998).

[36] T.K. Dey and S. Sen, Mod. Phys. Lett. A 23, 953 (2008).

[37] Amrita Bhattacharya and Alexander A. Potapov, Mod. Phys. Lett. A 29, 2399 (2010).

[38] T. Matsuda, Prog. Theor. Phys. 47, 738 (1972).

[39] J.A. Gonzalez, F.S. Guzman and O. Sarbach, Class. Quant. Grav. 26, 015010 (2009); ibid. 26, 015011 (2009). 